\title{
« De la musique, la littérature est le plus grand témoin ». Entretien avec Etienne Barilier
}

\section{Christophe Imperiali}

\section{(2) OpenEdition}

\section{Journals}

Édition électronique

URL : http://journals.openedition.org/edl/1160

DOI : $10.4000 /$ edl. 1160

ISSN : 2296-5084

Éditeur

Université de Lausanne

\section{Édition imprimée}

Date de publication : 15 décembre 2016

Pagination : 151-168

ISBN : ISBN 978-2-940331-50-5

ISSN : 0014-2026

Référence électronique

Christophe Imperiali, « « De la musique, la littérature est le plus grand témoin ». Entretien avec Etienne Barilier », Études de lettres [En ligne], 4 | 2016, mis en ligne le 15 décembre 2019, consulté le 15 décembre 2020. URL : http://journals.openedition.org/edl/1160; DOI : https://doi.org/10.4000/edl. 1160 


\title{
«DE LA MUSIQUE, LA LITTÉRATURE EST LE PLUS GRAND TÉMOIN".
}

\author{
ENTRETIEN AVEC ETIENNE BARILIER, \\ MENÉ PAR CHRISTOPHE IMPERIALI
}

Dans le cadre de la série de présentations dont le présent volume conserve la trace, Etienne Barilier avait proposé une conférence sur la Flûte enchantée de Mozart. Mais les éditeurs de ce volume ont eu envie de retrouver derrière le conférencier l'écrivain mélomane, dont l'œuvre est véritablement irriguée par un rapport intime à la musique. N'était-il pas pertinent, sans sortir de la liste des conférenciers, d'envisager un propos d'une autre nature et de proposer à Etienne Barilier un entretien sur la place que la musique occupe dans son œuvre? L'adhésion de l'intéressé à ce projet a donné lieu au présent article.

L'œuvre d'Etienne Barilier est constituée, pour l'heure, d'une cinquantaine de volumes, à peu près également répartis entre proses narratives et essais. De tout cet édifice, la première pierre a été posée en 1971, avec le roman Orphée. Ce titre programmatique plaçait d'emblée l'œuvre encore à venir sous la tutelle symbolique de celui qui est le père tout à la fois des poètes et des musiciens. Mais Orphée est aussi celui pour qui la question vitale est celle du regard en arrière. Aller de l'avant grâce à un passé nourricier, grâce à un dialogue fécond avec la culture occidentale, musicale et littéraire en particulier: tel est en effet le mouvement fondamental qui anime l'œuvre d'Etienne Barilier. Et dans cette culture qui fonde la substance de son écriture, la musique tient une place de choix. Plusieurs de ses textes, romans ou essais, parlent en effet 
de musique ${ }^{1}$. Mais en deçà des contenus, la musique innerve aussi plus profondément son imaginaire et son écriture elle-même.

Ce dialogue avec la culture occidentale est en outre le socle de méditations plus amères sur les déviances de notre civilisation. Paru entre l'entretien reproduit ci-dessous et la publication du présent volume, son dernier essai, Vertige de la force, s'attache à replacer les actes de barbarie qui frappent aujourd'hui l'Europe dans le cadre de cette culture qu'il croit capable de lui opposer une réponse humaniste. Le regard en arrière, ici, est salutaire, et ce sont les grands piliers de notre culture qu'il s'agit de garder à l'esprit pour ne pas sombrer. A ce titre, la musique et la littérature débordent largement la relation esthétique; elles fondent, pour Etienne Barilier, une véritable éthique, comme le montre la réponse qu’il donne à la première question ci-dessous.

Christophe Imperiali (CI) : Je me suis laissé dire que vous étiez un très bon pianiste, et que vous étiez même un peu compositeur, à vos heures... Quoi qu'il en soit, il n'est pas difficile de constater que votre connaissance de la musique est beaucoup plus intime que celle d'un simple mélomane. Quelle place la musique occupe-t-elle dans votre vie?

Etienne Barilier (EB) : Il se trouve que vos questions me parviennent peu après les attentats de Paris ${ }^{2}$, qui m’ont conduit à reprendre et à infléchir un essai intitulé Vertige de la force, qui va paraître en janvier 2016. Cela ne m'empêche pas de vous dire - au contraire, cela m'y pousse - que la musique occupe dans ma vie une place essentielle. Dans cet essai à paraître, j'évoque un fait bien connu, mais dont il ne faut pas se lasser de creuser la signification: les terroristes islamistes tuent des humains, mais dans le même geste meurtrier, ils s'en prennent aux œuvres d'art, aux spectacles ou aux livres, de Palmyre à Paris en passant par Bamyan, Tunis ou Tombouctou. Les dictatures haïssent les arts, chacun le sait. Mais pourquoi?

I. Le premier essai d'Etienne Barilier, en 1977, est consacré à Albert Camus; le second, l'année suivante, à Alban Berg: dès le début, les deux arts marchent main dans la main.

2. NDLR: L'entretien a été réalisé peu après les attentats qui ont frappé Paris en date du 13 novembre 2015. 
Non parce que les artistes seraient nécessairement des frondeurs et des rebelles par nature ou par vocation. Mais parce que l'œuvre d'art est toujours, dans le monde, le surgissement du neuf, c'est-à-dire de l'irréductible; à ce titre, elle est œuvre de liberté. Une fois tel quatuor de Mozart écrit, vous pouvez le rattacher à ce qui l'a précédé, dans l'histoire de la création mozartienne et l'histoire de la musique. Mais vous ne pouvez pas déduire ce quatuor de cette histoire. Vous ne pouvez pas réduire sa nouveauté. De même la liberté, dans la vie, est-elle ce qui nous permet de nous créer nous-mêmes, de faire advenir la nouveauté dans l'histoire humaine, c'est-à-dire de transcender les déterminismes, individuels et collectifs. Création et liberté sont synonymes. Sans doute, la liberté de l'œuvre d'art ne s'accomplit-elle pas dans nos vies concrètes. Nous la contemplons sans pouvoir nous en saisir dans nos existences. Mais tandis que nous la contemplons, elle nous donne l'idée, l'envie, la force de travailler à la liberté concrète.

J'ai conscience que cette conception de l'art est très platonicienne: l'œuvre d'art place devant nos yeux l'Idée de liberté, et cette Idée, sur les barricades du réel, guide le peuple de nos aspirations. Mais oui, je le crois, puisque les pires ennemis de la liberté le croient aussi, et tentent de détruire l'art pour mieux détruire notre espoir d'une humanité meilleure. Et pour revenir à la musique, elle occupe dans ma vie une place essentielle, mais elle ne sera jamais pour moi l'harmonieux moyen d'échapper au bruit et à la fureur du monde. Elle est me pousse au contraire à les affronter. Précisément parce qu'elle me propose et me donne de vivre une harmonie dont je veux intensément qu'elle se substitue, dans la réalité concrète, au règne du chaos.

$\mathrm{CI}$ : Avez-vous envisagé, à un moment ou à un autre, que la musique pourrait être votre "métier»?

EB : Non, mais je n'en ai pas moins rêvé, parfois, d'être musicien, et capable de transmettre à des auditeurs ces mélodies et ces harmonies qui sont peut-être le plus beau des mystères humains. Cependant, j'oubliais, dans ce rêve, que la musique, chez les interprètes les plus inspirés et les compositeurs les plus géniaux, est aussi un métier, comme vous le dites, et qu'il faut un labeur infini pour parvenir à l'immédiateté, à la simplicité qui est l'apanage des grands. Je ne suis pas un bon pianiste, hélas, et j’ai toujours voulu dévorer les partitions sans jamais les travailler, comme 
si je les sommais de se jouer toutes seules sous mes doigts, comme si je les vivais trop intensément pour qu'elles me refusent cette grâce. Leur liberté devait être mienne par une opération magique. Mais non, la liberté est un travail.

CI: La musique et les lettres sont-elles pour vous deux terrains distincts, ou plutôt deux faces d'un même objet? Et voyez-vous une différence de nature entre ce qui vous attire vers l'un et l'autre de ces arts?

EB: La musique et les lettres sont pour moi la face et le profil d'un visage aimé. Cette image me vient quand je songe à la difficulté où je suis de les séparer dans mon cœur et même dans mon esprit. Ce qui les rapproche le plus, c'est qu'il s'agit de deux arts du temps, deux arts qui nous font aimer, comprendre et peut-être, avec Marcel Proust, surmonter le temps. Dans sa Montagne magique, Thomas Mann observe que le temps de la littérature est très différent de celui de la musique parce que dans cette dernière, la durée de l'œuvre est en somme identique à la durée de ce qu'elle narre, tandis qu'un récit de quelques pages, et qu'on lit dans le laps de temps où l'on écoute une sonate, peut raconter un siècle ou une minute. Il y a donc, dans le temps littéraire, deux temps au moins. C'est assurément une différence profonde avec la musique. Ce dédoublement du temps n'est d'ailleurs pas sans rapport avec ce qu'on appelle la double articulation des mots de la littérature, par opposition à l'articulation simple des sons de la musique. En un sens, la littérature parle du monde, tandis que la musique parle le monde: elle est ce qu'elle dit. Et je ressens parfois cette immédiateté de la musique, cette plénitude ontologique, comme une forme de supériorité. Néanmoins, devant le temps comme mystère et comme destin, le temps de la vie et de la mort, musique et littérature sont sœurs. Elles sont là, toutes deux, pour le raconter, pour se l'approprier, pour en faire de la beauté.

CI: Face à l'idée wagnérienne que l'art le plus élevé résulterait de la fusion entre texte et musique, Mallarmé suggérait que, dans une telle fusion, ni l'un ni l'autre des arts ne donnait sa pleine mesure. Comment envisagez-vous ce partage?

EB: Le rêve de Wagner est celui d'une œuvre d'art totale où tous les arts (pas seulement le texte et la musique) conspirent à l'ivresse mystique. 
Mais la conjonction de plusieurs arts ne fait pas un art supérieur. Prenons L'Après-midi d'un faune de Mallarmé-Debussy-Nijinski: ce qui peut apparaittre comme trois versions d'une même œuvre (poésie, musique, danse) ne saurait en aucune manière s'additionner ni fusionner. Après avoir entendu l'œuvre de Debussy, Mallarmé avait eu cette magnifique phrase de dépit admiratif: "Le Faune, je croyais l'avoir moi-même mis en musique». Mais en somme il avait raison de le croire, comme il a raison dans la pensée que vous citez: ce Faune, il l'avait bel et bien mis en musique dans ses mots; la musique de Debussy, c'est tout simplement une autre œuvre, tout comme le sera l'interprétation chorégraphique de Nijinski (d'ailleurs ouvertement anti-debussyste).

La fusion des arts est un idéal qui, s'il fut jamais atteint, s'est irrémédiablement perdu dans le désenchantement du monde occidental. Pour l'Orient, la question se pose différemment: ne peut-on pas dire, par exemple, que dans la calligraphie chinoise, la peinture s'unit véritablement à l'écriture? En outre, dans la Chine traditionnelle, les sons de la musique correspondent aux planètes, aux heures, aux éléments, aux couleurs, aux passions de l'âme. On peut vraiment parler de correspondances au sens de Baudelaire. Mais pour revenir à l'Occident, le Gesamtkunstwerk est le rêve d'un paradis perdu, qui sans doute n'a jamais existé: l'opéra moderne n'est évidemment pas un retour à la tragédie grecque. Et l'envoûtement wagnérien n'est pas l'effet d'un art total redivivus, c'est l'envoûtement de la musique de Wagner.

CI: Dans le cycle de conférences que prolonge le présent volume, vous avez parlé de Mozart et de sa Flûte enchantée; vous avez en outre beaucoup écrit sur Wagner ou sur Berg en tant que compositeurs d'opéra. On a pourtant, à la lecture de votre œuvre, l'impression que l'opéra est un genre qui vous pose plus de problèmes que la musique de chambre, ou la musique pour piano...

EB : La cause en est peut-être futile: il me semble que la musique de chambre n'engendre pas d'auditeurs maniaques, alors que les opéramaniaques sont légion. Et chez ces derniers, bien souvent, on se passionne pour tout (voix, mise en scène, décors), sauf pour la musique. Dans leur esprit, la musique est moins maittresse que servante. Servante de leur passion pour les histoires de dieux du Wallhalla et de divas de la scène. Bien entendu, dans les grands opéras, et chez Mozart avant 
tout, la musique n'est pas moins sublime que dans un quatuor à cordes. Et je suis le premier à admirer sans réserve la puissance de révélation humaine et psychologique d'une grande musique associée à un livret de premier ordre (ceux de Da Ponte, de Busenello pour Monteverdi, de Hofmannstahl pour Strauss... ou celui que Berg a tiré de Büchner). Cela ne fait pas des œuvres d'art totales, mais de géniaux bouquets, et bien fou serait celui qui voudrait s'en priver.

CI : Dans le domaine de l'opéra, quelles sont vos prédilections? En dépit de votre goût pour l'Italie, vous parlez moins d'opéra italien que d'opéra allemand. Et de manière plus générale, il semble que le cœur de la culture que vous tenez pour vôtre est lié de près à la tradition germanique des $\mathrm{XIX}^{\mathrm{e}}$ et $\mathrm{XX}^{\mathrm{e}}$ siècle...

EB: Il n'est jamais trop tard pour s'enrichir: je viens de découvrir avec un ravissement certain L'élixir d'amour de Donizetti. Mais ma préférence va peut-être aux grands opéras de Mozart qui, à part la Flûte enchantée, sont chantés en italien. Ce qui unit, comme c'est mon vœu le plus cher, le sud et le nord de l'Europe. Il est vrai néanmoins que le cœur de la culture musicale se situe plutôt pour moi dans l'Europe septentrionale, tandis que le cœur de la culture plastique serait en Europe australe. Mais je suis toujours fasciné par les rencontres et les échanges, sans doute parce que je suis un enfant de l'Helvetia mediatrix. En peinture, Dürer découvrant Giovanni Bellini à Venise; en musique, à une tout autre époque, Busoni riche de son double héritage italo-germanique.

CI : Dans ce petit chef-d'œuvre qu'est Piano chinois, vous écrivez (ou plutôt: vous faites écrire à l'un de vos deux personnages) : «Nous vivons le temps de l'après-culture européenne» ${ }^{3}$. Il y a dans cette affirmation l'amorce de deux grands débats qui se trouvent au cœur de cet ouvrage (notamment) et que je voudrais aborder successivement; l'un est d'ordre spatial, l'autre d'ordre temporel.

Sur ce dernier plan, la question est celle de l'«après-culture». Vos textes sur la musique gravitent majoritairement autour de deux moments: le romantisme et la "modernité» du XXe siècle. Mais cette modernité est souvent située, de façon parfois implicite, dans une chro-

3. E. Barilier, Piano chinois, p. 111. 
nologie qui en fait un "après" - et en particulier un "après-romantisme». Dans votre roman Musique, c'est à partir d'un fictif compositeur contemporain, Joseph Kahn, que vous interrogez les catégories romantiques. Le jeune pianiste qui est le narrateur du récit est tourmenté, en particulier, par la question du génie:

Je m'intéresse à Joseph Kahn parce que je veux savoir si le génie est possible aujourd'hui. Je veux prouver qu'il est possible, et qu'on le nie par mesquinerie et par dépit ${ }^{4}$.

La musique moderne vous apparaît-elle comme la voix d'un paradis perdu, travaillée par la nécessité de continuer à s'exprimer (car l'expression est vitale) à un moment où l'on ne peut pourtant plus croire à l'expressivité telle qu'on l'envisageait un siècle plus tôt, où l'on ne peut plus croire à l'«inspiration» ou au "génie»?

EB : J'ai parfois l'impression que les grands compositeurs romantiques nous ont livré, une fois pour toutes, le chant parfait du cœur, le chant de la vie intérieure. Les lieder de Schubert disent d'une manière insurpassable l'amour de vivre et le désespoir de vivre. Mais aussi le piano de Chopin, celui de Schumann... Leur intériorité, n'est-ce pas exactement la nôtre, leur drame n'est-il pas le nôtre, leur angoisse et leur joie? Ils sont nos contemporains. Mais comment le compositeur d'aujourd'hui, qui voudrait aussi l'être, peut-il y parvenir? L'histoire de la musique à partir des années 1950, de l'abstraite sérialité généralisée à l'éclectisme et aux «retours à» qui sont le lot de notre aujourd'hui, me semble révélatrice. Les compositeurs contemporains les plus intéressants que je connaisse se refusent au pastiche et aux "retours à", mais leur musique, parfois d'une réelle beauté contemplative (je pense à George Benjamin, Toru Takemitsu, Elliott Carter), est souvent d'une extrême complexité, où menace de se dissoudre le chant de la vie intérieure. Le paradis perdu, c'est celui du temps où la complexité même s'épanouissait en simplicité: Berg a montré que la Rêverie de Schumann était une musique complexe. Et les lieder les plus nus de Schubert ne le sont guère moins. Mais c'est leur simplicité qui nous frappe et nous comble. Je ne dis pas qu'on n'y parviendra plus; mais à l'heure qu'il est, on n'y parvient guère.

4. Id., Musique, p. 85. 
CI : Peut-être est-ce cette impossibilité de reconduire aujourd'hui le discours romantique qui engage une forme de distanciation que l'on perçoit parfois derrière la ferveur qui vous lie à la musique romantique? Je pense notamment au traitement des personnages du Chien Tristan, ces musicologues qui vivent leur rapport aux compositeurs qu'ils étudient sur le mode de l'identification. Dans un tel cadre narratif, il est souvent difficile de déterminer si l'ironie qu'on sent poindre dans la narration porte sur le discours romantique lui-même, sur son idéologie, ses présupposés épistémologiques, ou plutôt sur le mode d'appropriation de ce discours par les scientifiques d'aujourd'hui...

EB: L'ironie ne visait pas, je crois, le romantisme lui-même (qui d'ailleurs fut souvent maître en ironie - je songe à Heinrich Heine), mais bien cette identification forcenée d'individus du $\mathrm{XX}^{\mathrm{e}}$ siècle à des compositeurs romantiques ou post-romantiques; c'est à coup sûr un geste désespéré vers le paradis perdu, et ne vont-ils pas, dans ce geste même de se retourner, comme Orphée, perdre leur Eurydice? Cependant, cette passion vaine, je la trouve légitime, respectable, émouvante, et mon ironie était affectueuse.

Votre question me fait aussi revenir à la précédente, et l'aborder sous un autre angle: considérons la totalité des musiques qui se font entendre aujourd'hui, que ce soit en concert ou par tous les médias possibles et imaginables: la proportion de celles qui ne sont pas tonales est infime. On patauge dans le tonalisme le plus bravement convenu, parfois le plus impudique, en tout cas le plus élémentaire. Or, qu'expriment-elles, ces musiques? L'amour, le temps qui passe, les rêves, les espoirs, les regrets, les chagrins, bref, les sentiments qui tissent notre vie à tous. Donc ce qu'exprimait Schubert, et qu'on ne peut se passer d'exprimer aujourd'hui - sauf qu'une pièce de Schubert est plus belle, plus riche, plus définitive qu'un million de tubes contemporains. Ce que je suis en train de dire, c'est que les romantiques ont trouvé (et souvent explicitement cherché, d'ailleurs) l'unité du savant et du populaire. Voilà sans doute le paradis que nous avons perdu: unir les richesses de l'esprit à la simplicité du cœur. Je ne dis pas qu'on ne puisse pas le retrouver dans le futur, mais il faudra beaucoup de génie pour éviter l'excès du «savant» (comme ce fut le cas à l'ère de la sérialité généralisée) comme celui du "populaire" (comme ce l'est parfois avec le «retour» à la tonalité). 
Bien sûr, on peut aussi estimer que la musique exprime et propose autre chose que ce que j'appelle le chant de la vie intérieure. On peut avoir, pour idéal, Bach plutôt que Chopin. Un idéal peut-être moins romantique, mais s'agirait-il pour autant d'un idéal de musique "objective», plus détachée des soucis de l'humanité? Bach lui-même était trop humain pour ne pas exprimer, notamment dans ses Passions, les sentiments les plus profonds. Le dernier chœur de la Passion selon saint Jean, cette berceuse au Christ mort, nous émeut profondément, même si ce n'est pas une émotion liée à des sentiments comme l'amour amoureux, ou l'angoisse individuelle devant la mort. Il me semble d'ailleurs que toute l'œuvre de Mendelssohn, avec cette retenue classique qu'on lui a reprochée, est le prolongement de cette émotion propre à Bach - ce Bach qu'il a tant contribué à faire revivre; le prolongement de cette tendresse contenue et maîtrisée qui n'en est que plus bouleversante. C'est dire aussi que de Bach au romantisme, comme en témoigne aussi Chopin (dont la Première Étude opus 10, pour ne nommer qu'elle, est le plus fulgurant et le plus puissant hommage qu'on ait jamais rendu à Bach), il n’y a pas solution de continuité. En revanche, après Schönberg, il y a rupture. Après lui, et non pas avec lui, qui garda toujours un profond souci d'expressivité.

CI : Un point sur lequel les deux interlocuteurs de Piano chinois semblent être plus ou moins d'accord est la menace que représente l'«ère numérique» pour la musique: l'un parle de «la fin des civilisations, au profit de l'universelle numérisation ${ }^{5}$, tandis que l'autre évoque le grouillement de l'humanité derrière les écrans d'ordinateurs, "milliards de mouches bourdonnantes sur le cadavre du silence - et de la musique» ${ }^{6}$. $\mathrm{La}$ «musique» est-elle vraiment en péril?

EB : A l'ère numérique, ce n'est pas seulement la musique, mais aussi les textes et les images qui sont en péril. Le danger de l'insignifiance par excès, cependant, n'est pas nouveau. Il a été dénoncé depuis longtemps, bien avant le règne d'Internet. Paul Valéry s'en alarmait il y a plus de quatre-vingts ans, et Goethe, encore cent ans plus tôt, parlait déjà d'un siècle "vélociférien». Disons simplement que la situation n'a fait

5. Id., Piano chinois, p. 111.

6. Ibid., p. 127. 
qu'empirer, et que la perte d'aura que Walter Benjamin déplorait à l'«ère de la reproductibilité technique» n’affecte pas seulement les images.

La situation a empiré, mais en même temps, elle se décante. Tout est à notre portée, et nous sommes contemporains de tout. Le moment est très proche, presque arrivé désormais, où tous les textes, toutes les images et toutes les musiques du monde nous seront disponibles instantanément. Les choses sont au moins claires: l'aura, c'est-à-dire la valeur unique et précieuse d'une œuvre, ne peut plus être éprouvée grâce à la distance, temporelle ou spatiale, qui nous en sépare; ni par l'effort de les atteindre (pour un tableau situé à l'autre bout du monde); ni par la rareté de leur exécution (pour une symphonie avant l'existence des enregistrements); ni par le choix que les relais, les médias, auraient opéré avant nous, en faveur de la qualité (pour la littérature). C'est notre discernement, notre silence intérieur, notre attention précise et fervente qui seuls peuvent retrouver l'aura des œuvres d'art.

Cela dit, les musées ne désemplissent pas, ni les salles de concert: le public sent bien que l'aura d'un tableau n'est jamais telle que devant ce tableau même; que l'aura d'une musique rayonne pleinement lorsque cette musique vit dans un concert. C'est peut-être, à cet égard, la littérature qui est la plus mal lotie, parce que le geste de sortir de chez soi, nécessaire à la contemplation du tableau et à l'écoute de la musique vivante, ne l'est pas pour la lecture d'un livre: "Je vais lire» ne signifie guère qu'on va se déplacer, en un lieu singulier et privilégié, pour lire. Aller au spectacle, c'est peu ou prou s'arracher au flux profane de la vie pour gagner un temple de l'art, musée ou salle de concert. «Aller lire» ne nous fait pas changer de cadre, et l'aura du livre doit briller dans l'espace de notre quotidien.

Tout cela me ramène à votre question sur la musique, à laquelle il me semble que la réponse doit être nuancée: contrairement à ce que disent mes personnages, la musique n'est probablement pas en péril dans l'univers numérique, du moins pas autant que la littérature.

CI : J'aimerais en venir maintenant à l'autre débat sous-jacent dans la notion d' "après-culture européenne": le débat spatial. La musique savante européenne peut-elle ou doit-elle être considérée comme un sommet artistique insurpassable, ou est-ce là une conception étroitement ethnocentrique? Les deux interlocuteurs que vous opposez sur cette question, dans Piano chinois, ont chacun des arguments forts à 
faire valoir, si bien qu'on ne sait trop sur quel pied danser... Avez-vous une position personnelle sur la question, ou pensez-vous qu'il est surtout important d'en formuler les termes finement pour susciter une réflexion?

EB : Le romancier, selon la formule consacrée, fait dialoguer les deux lobes de son cerveau. Et lorsqu'il met en scène des idées, ces idées deviennent des personnages. Ce qu'il y a d'unique dans le roman, c'est bien qu'il puisse donner chair à des idées opposées, comme il en va dans la vie. Il me semble en outre que dans le cas particulier que vous mentionnez - la musique savante européenne est-elle insurpassable, ou bien vaut-elle ni plus ni moins que toute autre musique, issue d'une autre civilisation ?- il n'y a évidemment pas de réponse "objective». La réponse dépend de ce qu'on attend de la musique, et de la définition qu'on aura donnée d'elle.

Après la parution d'une traduction japonaise de Piano chinois (j'ai eu le bonheur que ce livre soit traduit en plusieurs langues, et le bonheur tout particulier qu'il le soit en japonais et en chinois), j'ai été invité à faire des conférences au Japon, et $\mathrm{j}$ 'avais pris pour thème: «La musique européenne en Extrême-Orient». En préparant ces conférences, je suis tombé sur un texte que j'aurais peut-être intégré à mon livre s'il n'avait pas paru trop tard pour que je puisse en tenir compte. Ce texte a pour auteur Akira Mizubayashi, un écrivain japonais qui vécut longtemps en France et qui s'exprime directement en français, dans un ouvrage intitulé: Une langue venue d'ailleurs. A propos de son père qui écoutait, en pleine Deuxième Guerre mondiale, de la musique européenne, la musique de l'ennemi, Mizubayashi écrit :

Il tenait [...] comme à la prunelle de ses yeux à ce que [cette musique européenne] représentait: la modernité et la démocratie fondées sur la valeur suprême de l'individu.

Ce n'est pas moi, Européen, qui le dis, c'est un Japonais.

Oui, Beethoven ou Mozart ont écrit, aussi étrange que cela paraisse, une musique de la personne humaine. Car s'il est vrai, comme je l'avais relevé dans cette même conférence, que le compositeur européen distingue, plus que le musicien extrême-oriental, l'univers des sons musicaux de l'univers des murmures et des bruits naturels, n'est-ce pas qu'il donne à l'humain, dans sa spécificité, une place éminente et même suréminente? S'il est vrai que l'œuvre d'art, en Europe, est plus 
nettement détachée de la nature qu'en Extrême-Orient, et qu'elle tend d'une certaine manière à introduire dans le monde quelque chose que l'homme seul peut y créer, cela signifie bien que l'Europe accorde, à la personne humaine, une place singulière, et la plus haute de toutes.

On peut évidemment discuter cette suréminence de l'homme, et l'Europe elle-même ne s'est pas fait faute de s'auto-critiquer sur ce point, trouvant que l'homme "maître et possesseur de la nature» en était par trop le prédateur, etc. Cette question reste ouverte. Mais par-delà les opinions divergentes de mes personnages sur l'universalité de la musique européenne, je dirai que cette universalité ne relève évidemment pas du constat, mais du souhait. J'avoue alors que je partage ce souhait: je crois en effet qu'il y a une musique de la personne humaine, que Beethoven en est peut-être le plus extraordinaire représentant, et que si l'on croit à la personne humaine, si l'on veut la personne humaine, on aimera Beethoven par-dessus tout. Beethoven et quelques autres autour de lui, bien sûr.

Comment se fait-il que tel pianiste japonais m’ait confié qu'il trouvait ses «racines» dans Chopin et Debussy? Comment se fait-il que la ville de Shanghaï élève une statue à Chopin, et que la Chine, après les années terribles de la prétendue "révolution culturelle», se soit tournée avec une rare ferveur vers la musique classique occidentale? Ceci n'est évidemment pas sans rapport avec cela. Nous retrouvons ce mystère évident et irréductible de la relation - ou pour mieux dire, de la fraternité - entre art et liberté.

Vous le voyez, dans mes convictions personnelles, je ne garde pas la balance égale entre mes deux personnages. Mais j'espère que dans le roman, la balance est égale (et j'adhère d'ailleurs à certaines des critiques émises par celui qui ne croit pas à l'universalité de la musique occidentale). La conclusion du livre est ambiguë, comme est toujours la vie: la pianiste chinoise a-t-elle trouvé le secret de Chopin, ou bien a-t-elle compris comment on joue le secret de Chopin? Mais cette question, au fond, peut être posée à propos de n'importe quel interprète, fût-ce un Polonais qui joue Chopin, ou un Espagnol qui joue Albeniz. Qui sait s'il n'y a pas un paradoxe du pianiste comme il y a un paradoxe du comédien? Qu'est-ce qu'une expressivité authentique? Au-delà des interprètes, la même question peut être posée aux auditeurs - et, à tout seigneur tout honneur, aux compositeurs eux-mêmes: Nietzsche avait beaucoup à dire, vous le savez, sur Wagner l'envoûteur, le Cagliostro de la modernité... 
Pour revenir à la question de la musique européenne par rapport à d'autres musiques du monde, je ne crois pas à sa "supériorité», ou plutôt, ce n'est pas à sa supériorité que je crois. Cela n'aurait guère de sens. Je pense simplement que la musique occidentale, et l'art occidental en général, sont porteurs d'un certain idéal de la personne humaine, idéal qui me paraît digne d'être partagé par le monde entier. Cela n'empêche pas de goûter des musiques venues d'ailleurs, des musiques dont le sens même est profondément différent, mais tout aussi légitime.

Ce sens est peut-être, d'une manière noble et mystérieuse, un sens extra-humain; il ne consiste pas à construire musicalement la personne humaine, mais bien plutôt à se mettre à l'écoute du monde naturel, à descendre en lui, ou à le laisser descendre en soi. Claudel disait que la musique orientale est avant tout une manière de mieux écouter les murmures du monde, en leur donnant tout au plus, en toute délicatesse, une inflexion nouvelle. Cette musique, disait-il, rend sensible «le continu, cette présence indivisible hors de nous"; un poème japonais lui paraît semblable à une corde "où le musicien fait vibrer une seule note qui peu à peu envahit le cœur et la pensée». Autant d'expériences singulières, étrangères à notre tradition, mais qui peuvent puissamment nous enrichir - qui nous disent le monde, à nous qui disons l'homme.

CI: La relation dialectique qui s'instaure dans Piano chinois n'est pas une nouveauté dans votre œuvre. Musique reposait déjà sur l'antagonisme entre un pianiste et un critique, représentant deux conceptions fort différentes de la musique (encore que ce critique, comme son nom de plume "Florebius» le laisse entrevoir, soit lui-même marqué par une dualité interne). Et si l'on élargit un peu la perspective, on voit se dessiner un conflit récurrent entre deux grands types de rapport au monde: le rapport sensible et le rapport intellectuel (aux ouvrages ci-dessus, il faut ajouter au moins Le duel et Le rapt, où cette dualité est centrale). Mais vos sensibles sont parfois inadaptés, et vos intellectuels cyniques...

EB : Oui, je mets en scène le déchirement de ce que je voudrais voir uni: le sensible et l'intellectuel - ou l'intelligible. Je suis convaincu que l'intelligence est une forme de la sensibilité, et réciproquement. La compréhension intellectuelle du monde établit des rapports entre les choses, les soupèse, les compare, les éclaire l'une par l'autre; mais la compréhension sensible établit elle aussi des rapports entre les choses, des métaphores 
illuminantes. Bien sûr, l'approche intellectuelle ne peut pas supplanter l'approche sensible, ni l'approche sensible tenir lieu d'approche intellectuelle. L'homme a besoin des ouvres de l'intelligence comme il a besoin des œuvres de la sensibilité. Mais dans les grandes créations humaines, les deux approches se conjuguent, et c'est alors que nous sommes comblés. Je ne connais pas de grand artiste qui méprise l'intelligence ni de grand penseur qui soit fermé à toute sensibilité. Cependant, vous avez raison: dans mes livres, je mets souvent en scène des personnages chez qui l'intelligence et la sensibilité entretiennent des rapports "déséquilibrés». Je ne rêverais pas d'équilibre si je l'avais atteint.

CI: En musique, la dichotomie entre le sensible et l'intellectuel peut être saisie au moins à deux niveaux: celui de la composition et celui de la réception. Sur ce second plan, vous relevez au début de Piano chinois une vérité qui paraît rendre cette question incontournable pour tout auditeur averti :

[...] l'adolescent devient adulte. Les doigts des interprètes lui montrent la lune romantique, et de plus en plus souvent, il regarde les doigts. Il acquiert, avec la science de l'interprétation, le vice de la critique: ne plus lire le message, détailler le messager ${ }^{7}$.

Est-ce là une forme d'exil? Est-il fatal?

EB: Ce que je dénonce par la plume d'un de mes personnages me paraît bien réel, certes: comme dirait Monsieur de la Palice, un premier amour ne se vit qu'une fois; de même, la première écoute d'une œuvre musicale, la fraîcheur de la découverte, ne se retrouvera jamais. En outre, chez les auditeurs professionnels que sont les critiques musicaux, la routine s'installe. Et l'on tombe, à l'écoute d'un quatuor à cordes ou d'un pianiste, dans le vice que je reprochais aux opéramaniaques: on glose sur l'interprétation d'une œuvre qui semble, en soi, avoir perdu toute importance et tout intérêt. Elle est supposée connue, et pas même supposée aimée. Bref, on s'en tient résolument au second degré. Oui, ce danger est réel. D'un autre côté, c'est le privilège des grands interprètes de nous redonner une vision fraîche des œuvres, et, ajouterai-je, celui des grands auditeurs de revivre une œuvre, à n'importe quel âge de leur vie, comme

7. Ibid., p. 6. 
s'ils la découvraient, et d'y trouver des richesses qu'ils avaient sans doute éprouvées dans leur jeunesse, mais sans les mesurer pleinement. Donc les œuvres ne se fanent pas fatalement au fur et à mesure qu'on les réécoute et que le temps passe. Le contraire peut être vrai.

CI : Pour prolonger cette réflexion sur le sensible et l'intellectuel - ou sur la question du moyen de rendre intelligible un ressenti - citons un passage du Chien Tristan. Le personnage surnommé Schumann se met dans la peau du vrai Schumann, qui expose la finalité de sa démarche de compositeur: "c'est à cela qu'il faut en venir; à l'insertion, coûte que coûte, de ma musique intérieure dans le temps de notre humanité». Mais à ce défi du compositeur - transposer un mouvement intérieur en valeurs sonores, temporelles - s'ajoute celui que Schumann éprouve en glosant sa propre musique, puisque, dit-il, «le langage ne peut que rendre plus grossière la vérité de la musique, dont je parle à peu près comme je parlerais des sentiments si j'étais un homme froid: par la description de leurs effets les plus visibles ${ }^{8}$. Lorsqu'on lit cela sous la plume d'un romancier qui fait parler un musicologue incarnant un compositeur qui parle de sa musique, on mesure toute l'épaisseur de défi que représente cet objectif ultime de dire sa "musique intérieure»... Choisir les mots, serait-ce donc se couper de la possibilité même d'atteindre un tel objectif (pour autant que celui-ci soit partagé par l'écrivain)?

EB : Comme vous le pensez bien, et comme votre question le dit en creux, le romancier espère toujours que ses mots pourront saisir quelque chose de la «musique intérieure». Puisque c'est l'espoir même et le désir même d'en capter les mélodies et les harmonies qui, dès ses premiers essais d'adolescent, l'ont fait écrire! Si l'écrivain n'était pas animé par cet espoir, peut-être chimérique, il n'écrirait rien. De manière générale, il vit constamment dans ce paradoxe: il écrit parce qu'il éprouve que ce qu'il veut exprimer est indicible. Ce n'est même pas qu'il relève un défi. Mais l'impression est trop forte, l'appel trop puissant, la beauté trop impérieuse: il faut répondre. Toujours, l'indicible fait parler.

CI : Pour revenir à un aspect plus concret de la question des formes d'écriture: dans le livre qu'elle a consacré à la musique dans votre œuvre

8. E. Barilier, Le chien Tristan, p. 168. 
romanesque, Sylvie Jeanneret cherche à montrer que vous avez employé dans vos romans plusieurs formes empruntées à la musique. Elle évoque en particulier le leitmotiv, l'écriture contrapuntique et la structure des variations sur un thème. Voyez-vous dans de telles élaborations formelles une façon de rapprocher la prose romanesque de la musique? D'exploiter des procédés qui permettent de retremper l'écriture aux sources d'un art voisin, qui lui révélerait de nouvelles potentialités?

EB: Oui, il arrive que mes romans recourent à des modes d'écriture ou à des formes qu'on trouve dans la musique, et que vous indiquez. Parfois je l'ai fait consciemment, mais plus souvent encore, je crois, inconsciemment. De toute manière, les ressemblances, d'un art à l'autre, sont presque inévitables, même sans emprunt: encore une fois, la littérature et la musique sont des arts du temps, des arts d'organiser le temps; et quelles que soient les différences dans leur manière de s'y rapporter, on pourra trouver dans la construction d'un roman des structures, des formes, qui sont également présentes dans une symphonie. Et réciproquement. On a dit de Proust qu'il était le Wagner français. Ce n'est pas une vaine métaphore. Et Thomas Mann, justement lui, se définissait comme un musicien qui écrit des livres.

CI: Malgré tous les attraits que la musique présente à vos yeux, il me semble tout de même que ce n'est pas par hasard ou par défaut que vous avez choisi le langage des mots plutôt que celui des sons. N'êtes-vous pas trop préoccupé par le sens pour envisager de livrer une si grande part de son élaboration au récepteur? En tout cas, vos écrits sur la musique révèlent une préoccupation constante du sens de celle-ci - non pas simplement en tant que traduction en mots de ce qu'une musique pourrait représenter (comme lorsque votre Schumann glose ses Kreisleriana dans Le chien Tristan), mais en tant que "sens existentiel», pour reprendre une de vos expressions. Or ce sens existentiel, ne faut-il pas toutes les ressources du langage pour tracer autour de lui des cercles concentriques?

EB: Votre question en suppose une autre, qui peut-être déborderait notre propos: l'écoute d'une musique laisse-t-elle à l'auditeur une latitude d'interprétation plus grande que la lecture d'un roman? Peut-on faire, par exemple, une lecture anti-humaniste de Beethoven? Je n'en suis pas si sûr. Et même sans aller jusque-là, même si la musique est porteuse 
d'un sens qui ne se monnaie sans doute guère en mots, cela ne signifie pas que ce sens soit vague. C'est encore Mendelssohn qui disait que le sens de la musique n'était pas pour lui trop imprécis pour être exprimé par les mots, mais au contraire trop précis... Néanmoins, il serait vain de nier que le langage des mots entretient avec les concepts, les idées, et même simplement les objets du monde, une relation plus étroite que celle de la musique.

Mais pourquoi ai-je choisi le langage des mots? Est-ce à cause de cela? Comme il arrive toujours, on fait un choix, et si l'on en cherche les motifs, c'est beaucoup plus tard. Mon choix de l'écriture n'a pas été fait bien consciemment, ni par élimination d'autres modes d'expression. Mais peut-être en effet que ce choix peut trouver sa justification dans le besoin de sens dont vous parlez, et de sens formulable par le verbe.

Je disais tout à l'heure que l'immédiateté de la musique m’apparaissait comme un privilège refusé à la littérature. A cet égard, la musique est pour l'écrivain comme un paradis inaccessible, celui qu'il voudrait atteindre avec les mots, et qu'il n'atteindra jamais vraiment. Pourtant cette infériorité, cette insuffisance des mots, a pour envers, ou plutôt pour avers, un pouvoir que les mots sont les seuls à détenir: celui de nous donner l'intelligence du réel, sans pour autant nous priver de la beauté.

Certes, le langage est l'instrument des concepts, de la désignation précise des choses, de la démonstration; l'instrument du droit, des lois, des sciences de l'homme. Dès lors, n'est-ce pas un paradoxe et un privilège, avec cet instrument de l'intelligence, de pouvoir aussi créer des œuvres qui touchent et comblent les sens et la sensibilité, à l'égal de la peinture ou de la musique? L'ouvre d'art littéraire accomplie est aux prises avec la vérité, mais sa lutte et sa quête se font dans la lumière de la beauté. Le langage de la littérature est alors celui de l'intelligence rendue sensible au cœur, et celui d'un cœur qui jamais ne renonce à se faire comprendre. Oui, c'est sans doute pour cela que je l'aime, et que j'y recours - ou plutôt, que je m'y donne - pour écrire romans et essais, mais aussi pour dire, autant que je le puis, mon amour des autres arts, et ma dette à leur égard. De la musique, la littérature est le plus grand témoin.

Christophe IMPERIALI Université de Berne 


\section{BIBLIOGRAPHIE}

Bariller, Etienne, Orphée, Genève, Zoé, 1971.

—, Le chien Tristan, Lausanne, L'Age d'homme, 1977.

—, Alban Berg. Essai d'interprétation, Lausanne, L'Age d'homme, 1978.

—, Le rapt, Lausanne, L'Age d'homme, 1980.

—, Le duel, Lausanne, L'Age d'homme, 1983.

—, Musique, Lausanne, L’Age d'homme, 1988.

—, Piano chinois, Genève, Zoé, 2011.

—, Vertige de la force, Paris, Buchet/Chastel, 2016.

Jeanneret, Sylvie, La musique dans l'auvre romanesque d'Etienne Barilier. Vers une poétique de la modernité, Genève, Slatkine, 1998. 